\title{
SUBSPACES INTERSECTING IN AT MOST A POINT
}

\author{
SASCHA KURZ
}

\begin{abstract}
We improve on the lower bound of the maximum number of planes in $\mathrm{PG}(8, q) \cong \mathbb{F}_{q}^{9}$ pairwise intersecting in at most a point. In terms of constant dimension codes this leads to $A_{q}(9,4 ; 3) \geq q^{12}+2 q^{8}+$ $2 q^{7}+q^{6}+2 q^{5}+2 q^{4}-2 q^{2}-2 q+1$. This result is obtained via a more general construction strategy, which also yields other improvements.

Keywords: constant dimension codes, finite projective geometry, network coding

MSC: Primary 51E20; Secondary 05B25, 94B65.
\end{abstract}

\section{INTRODUCTION}

Let $V \cong \mathbb{F}_{q}^{v}$ be a $v$-dimensional vector space over the finite field $\mathbb{F}_{q}$ with $q$ elements. We call each $k$-dimensional linear subspace of $V$ a $k$-space, also using the terms points, lines, and planes for 1-, 2 -, and 3-spaces, respectively. Two $k$-spaces $U, W$ are said to trivially intersect or to be disjoint if $\operatorname{dim}(U \cap W)=0$, i.e., $U$ and $W$ do not share a common point. Sets of $k$-spaces that are pairwise disjoint are called partial $k$-spreads, see [10] for a recent survey on bounds for their maximum possible sizes. In finite projective geometry they are a classical topic. Here we study the rather similar objects of sets of $k$ spaces which pairwise intersect in at most a point and have large cardinality. More generally, we can use the subspace distance $\mathrm{d}_{\mathrm{s}}(U, W)=\operatorname{dim}(U+W)-\operatorname{dim}(U \cap W)=\operatorname{dim}(U)+\operatorname{dim}(W)-2 \operatorname{dim}(U \cap W)$ to define $A_{q}(v, d ; k)$ as the maximum number of $k$-spaces in $\mathbb{F}_{q}^{v}$ that have minimum subspace distance $d$, i.e., that intersect in a subspace of dimension at most $k-d / 2$. Since those sets, which are also called constant dimension codes, have applications in error correcting random network coding, see e.g. [11], bounds for $A_{q}(v, d ; k)$ have been studied intensively in the literature. For the currently best known lower and upper bounds we refer to the online tables http:// subspacecodes.uni-bayreuth. de and the associated survey [7]. Due to this connection, we also call sets of $k$-spaces codes and call their elements codewords.

Due to combinatorial explosion, it is in general quite hard to obtain improvements for $A_{q}(v, d ; k)$ when the dimension $v$ of the ambient space is small, say $v \leq 11$. Our main motivation for this paper is the recently improved parametric lower bound $A_{q}(9,4 ; 3) \geq q^{12}+2 q^{8}+2 q^{7}+q^{6}+q^{5}+q^{4}+1$, see [2, Theorem 3.13]. Here, we give a further improved construction for $A_{q}(9,4 ; 3)$ and generalize the underlying ideas to a more general combination of constant dimension codes. The latter constitutes our main Theorem, see Theorem 3, which allows to conclude also other improved parametric constructions.

\section{PRELIMINARIES}

For two matrices $U, W \in \mathbb{F}_{q}^{m \times n}$ we define the rank distance $\mathrm{d}_{\mathrm{r}}(U, W):=\operatorname{rk}(U-W)$. A subset $\mathcal{C} \subseteq \mathbb{F}_{q}^{m \times n}$ is called a rank metric code.

Theorem 1. (see [4]) Let $m, n \geq d$ be positive integers, $q$ a prime power, and $\mathcal{C} \subseteq \mathbb{F}_{q}^{m \times n}$ be a rank metric code with minimum rank distance $d$. Then, $\# \mathcal{C} \leq q^{\max \{n, m\} \cdot(\min \{n, m\}-d+1)}$.

Codes attaining this upper bound are called maximum rank distance (MRD) codes. They exist for all choices of parameters. A construction can e.g. be described using so-called linearized polynomials, see e.g. [11, Section V]. If $m<d$ or $n<d$, then only $\# \mathcal{C}=1$ is possible, which can be achieved by a zero 
matrix and may be summarized to the single upper bound $\# \mathcal{C} \leq\left\lceil q^{\max \{n, m\} \cdot(\min \{n, m\}-d+1)}\right\rceil$. Using an $m \times m$ identity matrix as a prefix one obtains the so-called lifted MRD codes.

Theorem 2. [13, Proposition 4] For positive integers $k, d, v$ with $k \leq v, d \leq 2 \min \{k, v-k\}$, and deven, the size of a lifted MRD code in $\left[\begin{array}{l}V \\ k\end{array}\right]$ with subspace distance dis given by $q^{\max \{k, v-k\} \cdot(\min \{k, v-k\}-d / 2+1)}$.

\section{COMBINING SUBSPACES}

Theorem 3. Let $\mathcal{C}_{1}$ be a set of $k$-spaces in $\mathbb{F}_{q}^{v_{1}}$ mutually intersecting in at most a point, $\mathcal{C}_{1}^{C}$ be a subset of $\mathcal{C}_{1}$ such that all elements are pairwise intersecting trivially, and $\mathcal{C}_{2}$ be a set of $k$-spaces in $\mathbb{F}_{q}^{v_{2}}$ mutually intersecting in at most a point, where $v_{2} \geq 2 k$ and $\# \mathcal{C}_{2} \geq 1$. If $\mathbb{F}_{q}^{v_{2}}$ admits a $\left(v_{2}-k\right)$-space $S$, such that exactly $\Lambda$ elements of $\mathcal{C}_{2}$ are contained in $S$ and all others intersect $S$ in at most a point, then

$$
A_{q}\left(v_{1}+v_{2}-k, 2 k-2 ; k\right) \geq \# \mathcal{C}_{1} \cdot q^{2\left(v_{2}-k\right)}+\# \mathcal{C}_{1}^{C} \cdot\left(\# \mathcal{C}_{2}-q^{2\left(v_{2}-k\right)}-\Lambda\right)+\Lambda .
$$

Proof. We embed $\mathcal{C}_{1}$ in $\mathbb{F}_{q}^{v_{1}+v_{2}-k}$ and choose a $\left(v_{2}-k\right)$-space $S$ disjoint to the span $\left\langle\mathcal{C}_{1}\right\rangle$. For each $U \in \mathcal{C}_{1}$ we consider the $v_{2}$-space $K=\langle U, S\rangle$. If $U \in \mathcal{C}_{1}^{C}$, we embed $\mathcal{C}_{2}$ minus the $\Lambda$ codewords contained in $S$ in $K$ such that the embedding contains the $k$-space $U$ and all codewords intersect $S$ in at most a point. If $U \notin \mathcal{C}_{1}^{C}$, we embed a lifted MRD code in $K$ such that the embedding contains the $k$-space $U$ and all codewords are disjoint to $S$. If we additionally add $\Lambda$ codewords inside $S$, then we obtain a set $\mathcal{C}$ of $k$-spaces in $\mathbb{F}_{q}^{v_{1}+v_{2}-k}$ of cardinality $\# \mathcal{C}_{1}^{C} \cdot\left(\# \mathcal{C}_{3}-\Lambda\right)+\left(\# \mathcal{C}_{1}-\# \mathcal{C}_{1}^{C}\right) \cdot q^{2\left(v_{2}-k\right)}+\Lambda$, since the matching lifted MRD code has cardinality $q^{2\left(v_{2}-k\right)}$. For two different $W, W^{\prime} \in \mathcal{C}$ we have to show that they do intersect in at most a point. By construction, there exist $U, U^{\prime} \in \mathcal{C}_{1}$ such that $W \leq K:=\langle U, S\rangle$ and $W^{\prime} \leq K^{\prime}:=\left\langle U^{\prime}, S\right\rangle$. We have $S \leq K \cap K^{\prime}$ and $v_{2}-k \leq \operatorname{dim}\left(K \cap K^{\prime}\right)=v_{2}-k+\operatorname{dim}\left(U \cap U^{\prime}\right) \leq$ $v_{2}-k+1$. If $U=U^{\prime}$, which we can assume w.l.o.g. for $W \leq S$ or $W^{\prime} \leq S$, then $\operatorname{dim}\left(W \cap W^{\prime}\right) \leq 1$. If $U, U^{\prime} \in \mathcal{C}_{1}^{C}$, then $W \cap W^{\prime} \leq S$, so that $\operatorname{dim}\left(W \cap W^{\prime}\right) \leq 1$. Otherwise we have $\operatorname{dim}\left(W \cap W^{\prime} \cap S\right)=0$, so that also $\operatorname{dim}\left(W \cap W^{\prime}\right) \leq 1$.

If we choose $v_{2}=2 k$ and $\mathcal{C}_{2}$ such that there are two disjoint codewords, then $S$ can be chosen as a codeword, i.e., $\Lambda=1$, and all codewords except $S$ itself intersect $S$ in at most a point. For brevity, we will calls sets of $k$-spaces that are trivially intersecting and are a subset of a some set $\mathcal{C}_{1}$ of $k$-spaces, a clique.

\section{Corollary 4.}

$$
A_{q}(9,4 ; 3) \geq q^{12}+2 q^{8}+2 q^{7}+q^{6}+2 q^{5}+2 q^{4}-2 q^{2}-2 q+1
$$

PROOF. For $k=3$ and $v=6$ we choose $\mathcal{C}_{1}$ and $\mathcal{C}_{2}$ as a set of $q^{6}+2 q^{2}+2 q+1$ planes in $\mathbb{F}_{q}^{6}$ pairwise intersecting in at most a point [3, Theorem 2.1]. By [2, Theorem 3.12] we can choose a subset $\mathcal{C}_{1}^{C} \subseteq \mathcal{C}_{1}$ of cardinality $q^{3}-1$.

We remark that this improves the very recent lover bound $A_{q}(9,4 ; 3) \geq q^{12}+2 q^{8}+2 q^{7}+q^{6}+q^{5}+q^{4}+1$ [2. Theorem 3.13]. As $\mathcal{C}_{2}$ we might also have chosen the construction from [9] of the same size ${ }^{1}$ In our setting we always have $\# \mathcal{C}_{1}^{C} \leq A_{q}(6,6 ; 3)=q^{3}+1$. If we replace $\mathcal{C}_{2}$ in Corollary 4 by the set of $q^{8}+q^{5}+q^{4}-q-1$ planes in $\mathbb{F}_{q}^{7}$ from [8, Theorem 3], then the conditions of Theorem 3 are satisfied for $\Lambda=0$ and we obtain

$$
A_{q}(10,4 ; 3) \geq q^{14}+2 q^{10}+2 q^{9}+2 q^{8}+q^{7}-q^{5}-2 q^{4}-q^{3}+q+1 .
$$

However, [12, Proposition 4.4] gives a better lower bound.

For a general application of Theorem 3 the presumably hardest part is to analytically determine $\mathcal{C}_{1}^{C}$, i.e., a clique in $\mathcal{C}_{1}$. If $\mathcal{C}_{1}$ itself is obtained via Theorem 3 and a lower bound on the clique size of the corresponding part $\mathcal{C}_{2}$ is known, then can recursively determine suitably large cliques.

\footnotetext{
${ }^{1}$ The same applies to $\mathcal{C}_{1}$, i.e., we can avoid to use [2. Theorem 3.12], see the subsequent Footnote 3
} 
Lemma 5. If $\mathcal{C}$ is obtained from the construction of Theorem 3 and the corresponding part $\mathcal{C}_{2}$ contains a clique $\mathcal{C}_{2}^{C}$ whose elements are disjoint from $S$, then $\mathcal{C}$ admits a subset $\mathcal{C}^{\prime}$ such that all elements are pairwise intersecting trivially and $\# \mathcal{C}^{\prime}=\# \mathcal{C}_{1}^{C} \cdot \# \mathcal{C}_{2}^{C}$.

Proof. Using the notation from Theorem 3 we construct $\mathcal{C}^{\prime}$. For each $U \in \mathcal{C}_{1}^{C}$ we consider $K:=\langle U, S\rangle$ and choose a clique of cardinality $\# \mathcal{C}_{2}^{C}$ in $K$ and add the elements to $\mathcal{C}^{\prime}$. Using the analysis of the proof of Theorem 3 again and the fact that the elements of $\mathcal{C}^{\prime}$ all are disjoint to $S$, we conclude that the elements of $\mathcal{C}^{\prime}$ are pairwise intersecting trivially.

If we choose $\mathcal{C}_{2}$ according to [3, Theorem 2.1], we can use [2, Theorem 3.12] to conclude $\# \mathcal{C}_{2}^{C} \geq$ $q^{3}-1$.

Proposition 6. $A_{q}(6+3 t, 4 ; 3) \geq\left(q^{6}+2 q^{2}+2 q+1\right) \cdot q^{6 t}+\frac{q^{6 t}-1}{q^{6}-1}+\sum_{i=1}^{t}\left(2 q^{2}+2 q\right) \cdot\left(q^{3}-1\right)^{i} \cdot q^{6(t-i)}$ for all $t \geq 0$.

Proof. For the induction start $t=0$ we choose $\mathcal{C}^{(0)}$ as a set of $q^{6}+2 q^{2}+2 q+1$ planes in $\mathbb{F}_{q}^{6}$ pairwise intersecting in at most a point according to [3, Theorem 2.1], which admits a clique of cardinality $q^{3}-1$. For the induction step $\mathcal{C}^{(i)} \rightarrow \mathcal{C}^{(i+1)}$ we apply Theorem 3 with $v_{2}=2 k, \Lambda=1, \mathcal{C}_{1}=\mathcal{C}^{(i)}$, and $\mathcal{C}_{2}=\mathcal{C}^{(0)}$. By induction, see Lemma $5, \mathcal{C}^{(i)}$ admits a clique $\mathcal{C}_{1}^{C}$ of cardinality $\left(q^{3}-1\right)^{i+1}$. The induction hypothesis for the cardinality of $\mathcal{C}^{(i)}$ is

$$
\# \mathcal{C}^{(i)}=\left(q^{6}+2 q^{2}+2 q+1\right) \cdot q^{6 i}+\frac{q^{6 i}-1}{q^{6}-1}+\sum_{j=1}^{i}\left(2 q^{2}+2 q\right) \cdot\left(q^{3}-1\right)^{i} \cdot q^{6(i-j)}
$$

and the induction step, see Theorem 3, gives $\# \mathcal{C}^{(i+1)}$ as the right hand side of Equation 22, where $i$ is replaced by $i+1$.

Another example of a set of planes pairwise intersecting in at most a point, where we can analytically determine a reasonably large clique, is given by [12, Proposition 4.4]: $A_{q}(8,4 ; 3) \geq q^{10}+q^{6}+q^{5}+$ $2 q^{4}+2 q^{3}+2 q^{2}+q+1$, which is the currently best known lower bound for $q \geq 3$. The essential key here is that the code contains a lifted MRD code of cardinality $q^{10}$ for rank distance 2 . By [5, Lemma 5] the MRD code can be chosen in such a way that it contains a subcode of cardinality $q^{5}$ and rank distance $32^{2}$ Thus we obtain a clique of cardinality $q^{5}$ and can use Theorem 3 with $v_{2}=6$ and $\Lambda=1$ to conclude

$$
A_{q}(11,4 ; 3) \geq q^{16}+q^{12}+q^{11}+2 q^{10}+2 q^{9}+2 q^{8}+2 q^{7}+2 q^{6}+1,
$$

which strictly improves upon [12, Proposition 4.4]. Of course we can iteratively apply the combination with the $q^{6}+2 q^{2}+2 q+1$ planes in $\mathbb{F}_{q}^{6}$ to obtain an infinite parametric series as in Proposition 6 . The method generalizes to cases where large constant dimension codes are obtained by using lifted MRD codes as subcodes, which frequently is the case. Also the constant dimension codes showing $A_{q}(6,4 ; 3) \geq q^{6}+2 q^{2}+2 q+1$ [9. Lemma 12, Example 4] and $A_{q}(7,4 ; 3) \geq q^{8}+q^{5}+q^{4}+q^{2}-q$ [8. Theorem 4] are closely related. They both arise by starting from a lifted MRD code, removing some planes, and then extending again with a larger set of planes, cf. [1]. Considering just the reduced lifted MRD code, we can deduce clique sizes of $q^{3}-1$ and $q^{4}$, respectively ${ }^{3}$ If we choose $\mathcal{C}_{1}$ in Theorem 3 as the mentioned code for $A_{q}(7,4 ; 3)$ and $\mathcal{C}_{2}$ as the mentioned code for $A_{q}(6,4 ; 3)$ or the code for

\footnotetext{
${ }^{2}$ Using linearized polynomials to described the lifted MRD code, a clique of matching size can be described as the set of monomials $a x$ (including the zero polynomial).

${ }^{3}$ Both constructions are stated in the language of linearized polynomials. For [9] Lemma 12, Example 4] the representation $\mathbb{F}_{q}^{6} \cong \mathbb{F}_{q^{3}} \times \mathbb{F}_{q^{3}}$ is used and the planes removed from the lifted MRD code correspond to $u x^{q}-u^{q} x$ for $u \in \mathbb{F}_{q^{3}}$, so that the monomials $a x$ for $a \in \mathbb{F}_{q^{3}} \backslash\{\mathbf{0}\}$ correspond to a clique of cardinality $q^{3}-1$. For 8 . Theorem 4] the representation $\mathbb{F}_{q}^{7} \cong W \times \mathbb{F}_{q^{4}}$, where $W$ denotes the trace-zero subspace of $\mathbb{F}_{q^{4}} / \mathbb{F}_{q}$, is used. The planes removed from the lifted MRD code correspond to $r\left(u x^{q}-u^{q} x\right)$ for $r \in \mathbb{F}_{q^{4}} \backslash\{\mathbf{0}\}$ and $u \in \mathbb{F}_{q^{4}}$ with $\operatorname{tr}(u)=1$, so that the monomial s $a x$ for $a \in \mathbb{F}_{q^{4}}$ correspond to a clique of cardinality $q^{4}$.
} 
$A_{q}(7,4 ; 3) \geq q^{8}+q^{5}+q^{4}-q-1$, see[8, Theorem 3], then we obtain

$$
A_{q}(10,4 ; 3) \geq q^{14}+q^{11}+q^{10}+q^{8}-q^{7}+2 q^{6}+2 q^{5}+1
$$

and

$$
A_{q}(11,4 ; 3) \geq q^{16}+q^{13}+q^{12}+q^{10}+q^{8}-q^{5}-q^{4} .
$$

Both inequalities improve upon the (for $q \geq 4$ ) previously best known lower bounds from [12, Proposition 4.4] and the latter improves upon Inequality (3).

So, Theorem 3 can yield improved constructions, but of course not all choices of the involved parameters and codes lead to improvements. If $v_{1}<2 k$, then $\# \mathcal{C}_{1}^{C} \leq 1$, so that no strict improvement over known constructions can be obtained. For $k>3$ it might be necessary to use $v_{2}>2 k$, since no example for $A_{q}(2 k, 2 k-2 ; k)>q^{2 k}+1$ is known. In [6] the authors have indeed shown $A_{2}(8,6 ; 4)=2^{8}+1=257$ and conjectured $A_{q}(2 k, 2 k-2 ; k)=q^{2 k}+1$ for all $k \geq 4$.

In principle it is also possible to generalize Theorem 3 to situations where the $k$-spaces can intersect in subspaces of dimension $t$ strictly larger than one. To this end, one may partition $\mathcal{C}_{1}$ into subsets $\mathcal{C}_{1}^{(0)}, \mathcal{C}_{1}^{(1)}$, $\ldots, \mathcal{C}_{1}^{(t)}$ such that every element from $\mathcal{C}_{1}^{(i)}$ intersects each different element from $\cup_{j=0}^{i} \mathcal{C}_{1}^{(j)}$ in dimension at most $i$, which generalizes the partition $\mathcal{C}_{1}^{C}, \mathcal{C}_{1} \backslash \mathcal{C}_{1}^{C}$. If $S$ is again our special subspace and $U \in \mathcal{C}_{1}^{(i)}$, then codewords in the code in $\langle U, S\rangle$ should intersect $S$ in dimension at most $t-i$, where we may also put some additional codewords into $S$. Since we currently have no example at hand that improves upon a best known lower bound for $A_{q}(v, d ; k)$, we refrain from giving a rigorous proof and detailed statement.

\section{ACKNOWLEDGMENT}

The author would like to thank Thomas Honold for his analysis of possible cliques sizes in the constant dimension codes from [9, Lemma 12, Example 4] and [8, Theorem 4], see Footnote 3. The main idea for Theorem 3 is inspired by [2].

\section{REFERENCES}

[1] J. Ai, T. Honold, and H. Liu. The expurgation-augmentation method for constructing good plane subspace codes. arXiv preprint 1601.01502, 2016.

[2] A. Cossidente, G. Marino, and F. Pavese. Subspace code constructions. arXiv preprint 1905.11021, 2019.

[3] A. Cossidente and F. Pavese. On subspace codes. Designs, Codes and Cryptography, 78(2):527-531, 2016.

[4] P. Delsarte. Bilinear forms over a finite field, with applications to coding theory. Journal of Combinatorial Theory, Series A, 25(3):226-241, 1978.

[5] T. Etzion and N. Silberstein. Codes and designs related to lifted MRD codes. IEEE Transactions on Information Theory, 59(2):1004-1017, 2013.

[6] D. Heinlein, T. Honold, M. Kiermaier, S. Kurz, and A. Wassermann. Classifying optimal binary subspace codes of length 8, constant dimension 4 and minimum distance 6. Designs, Codes and Cryptography, 87(2-3):375-391, März 2019.

[7] D. Heinlein, M. Kiermaier, S. Kurz, and A. Wassermann. Tables of subspace codes. arXiv preprint 1601.02864, 2016.

[8] T. Honold and M. Kiermaier. On putative $q$-analogues of the Fano plane and related combinatorial structures. In Dynamical systems, number theory and applications, pages 141-175. World Sci. Publ., Hackensack, NJ, 2016.

[9] T. Honold, M. Kiermaier, and S. Kurz. Optimal binary subspace codes of length 6, constant dimension 3 and minimum subspace distance 4. In Topics in finite fields, volume 632 of Contemp. Math., pages 157-176. Amer. Math. Soc., Providence, RI, 2015.

[10] T. Honold, M. Kiermaier, and S. Kurz. Partial spreads and vector space partitions. In Network Coding and Subspace Designs, Signals and Communication Technology, pages 131-170. Springer, Cham, Januar 2018.

[11] R. Kötter and F. R. Kschischang. Coding for errors and erasures in random network coding. IEEE Transactions on Information Theory, 54(8):3579-3591, 2008.

[12] S. Kurz. A note on the linkage construction for constant dimension codes. arXiv preprint 1906.09780, 2019.

[13] D. Silva, F. Kschischang, and R. Kötter. A rank-metric approach to error control in random network coding. IEEE Transactions on Information Theory, 54(9):3951-3967, 2008.

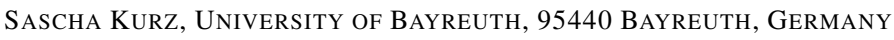

Email address: sascha.kurz@uni-bayreuth.de 